Supporting Information

\title{
Heterovalent Cation Substitution to Enhance the Ionic Conductivity of Halide Electrolytes
}

\author{
Xuming $\mathrm{Luo}^{\mathrm{a}}$, Xianzhang $\mathrm{Wu}^{\mathrm{b}}$, Jiayuan Xiang ${ }^{\mathrm{b}}$, Dan $\mathrm{Cai}^{\mathrm{a}}$, Min $\mathrm{Li}^{\mathrm{b}}$, Xiuli \\ Wang ${ }^{\mathrm{a} *}$, Xinhui Xia ${ }^{\mathrm{a}}$, Changdong $\mathrm{Gu}^{\mathrm{a}}$, Jiangping $\mathrm{Tu}^{\mathrm{a}}$

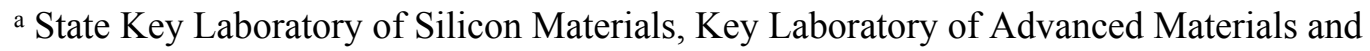 \\ Applications for Batteries of Zhejiang Province, and School of Materials Science and \\ Engineering, Zhejiang University, Hangzhou 310027, China. \\ ${ }^{\mathrm{b}}$ Narada Power Source Co., Ltd, Hangzhou 311305, China, Narada Ess Integration \& \\ Operation Co., Ltd Hangzhou 310012, China \\ * Corresponding author. Tel.: +86 571 87952573; Fax: +86 57187952856. \\ E-mail address:wangxl@zju.edu.cn;
}




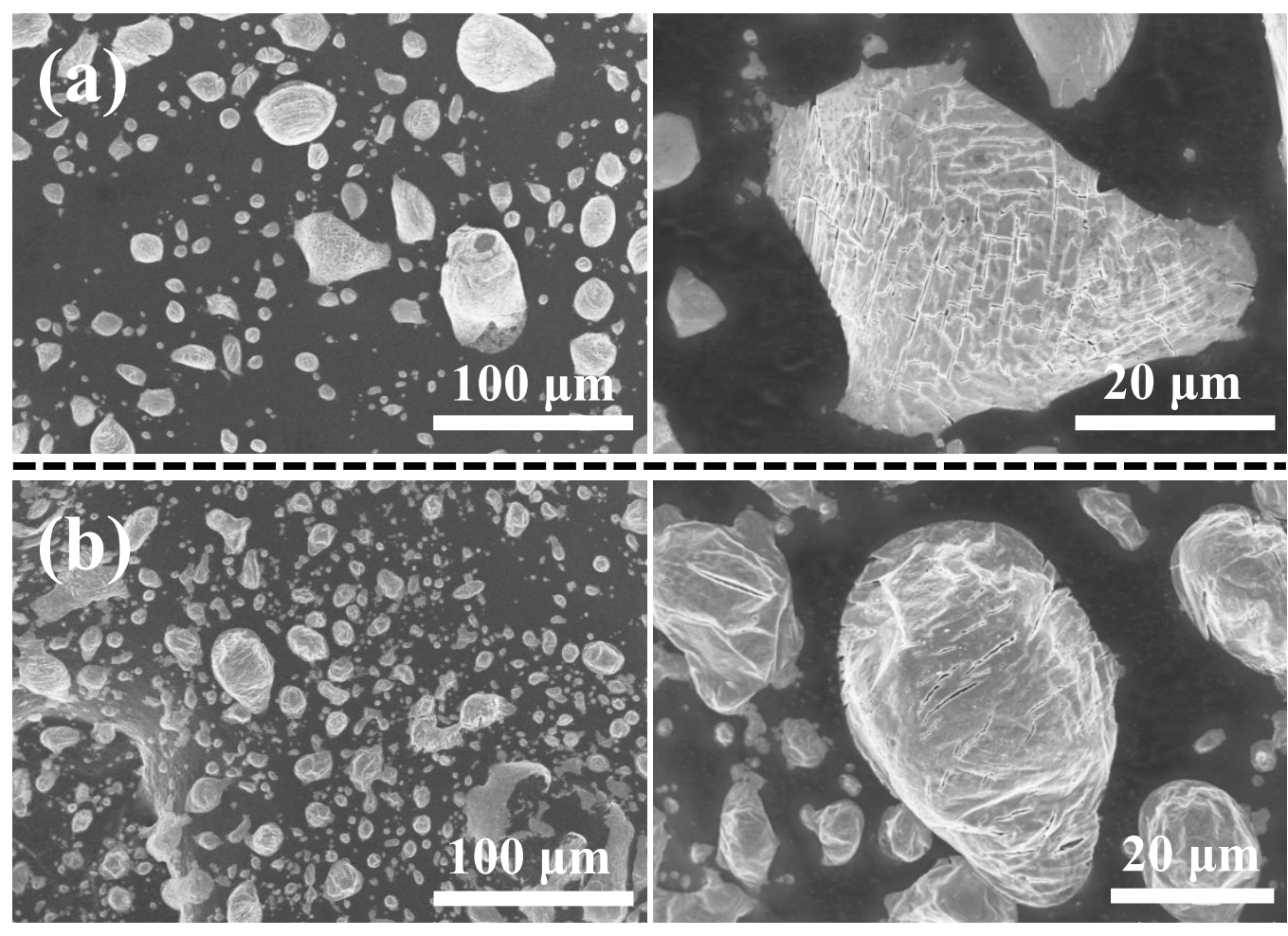

Figure S1 The micro-morphology of prepared halide $\mathrm{Li}_{3-\mathrm{x}} \mathrm{In}_{1-\mathrm{x}} \mathrm{Zr}_{\mathrm{x}} \mathrm{Cl}_{6}$ particles when (a) $\mathrm{x}=0$ and

(b) $x=0.1$ 


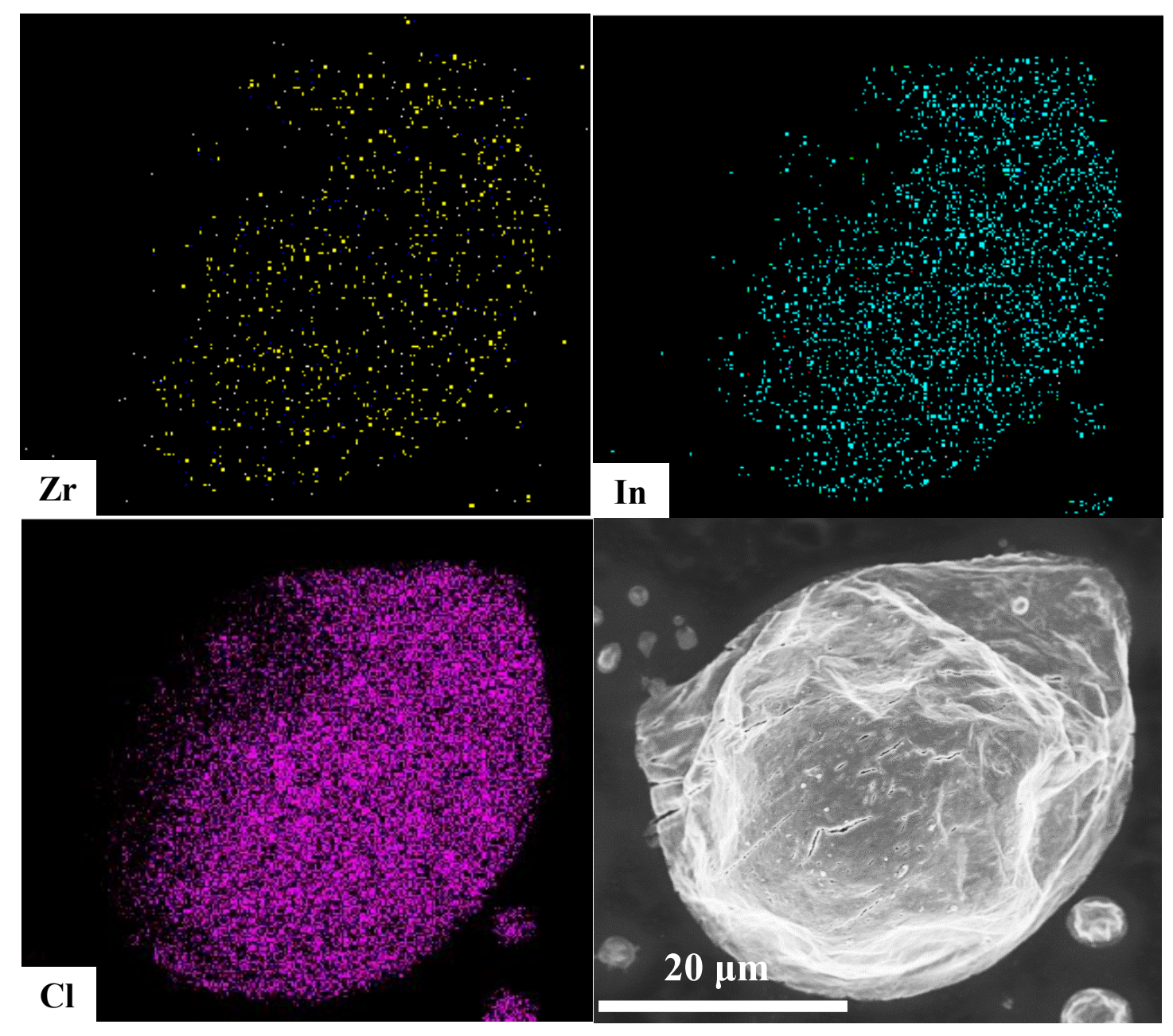

Figure S2 EDS mapping of prepared $\mathrm{Li}_{3-\mathrm{x}} \mathrm{In}_{1-\mathrm{x}} \mathrm{Zr}_{\mathrm{x}} \mathrm{Cl}_{6}(\mathrm{x}=0.1)$ halides particle.

Table S1 The ionic conductivity of halide solid electrolytes in previous work and this work.

\begin{tabular}{|c|c|c|}
\hline Compound & Ionic conductivity $/ \mathrm{S} \mathrm{cm}^{-1} @ 25^{\circ} \mathrm{C}$ & Reference \\
\hline LiX & $10^{-7}$ & $1-3$ \\
\hline $\mathrm{LiAlCl}_{4}$ & $10^{-6}$ & $4-5$ \\
\hline $\mathrm{Li}_{\mathrm{x}} \mathrm{M}_{\mathrm{y}} \mathrm{F}(\mathrm{M}=\mathrm{Mg}, \mathrm{Pb}$, etc $)$ & $10^{-8}-10^{-6}$ & $6-8$ \\
\hline $\mathrm{Li}_{3} \operatorname{InBr}_{6}$ & $10^{-7}$ & 9 \\
\hline $\mathrm{Li}_{3} \mathrm{YCl}_{6}$ & $0.51 \times 10^{-3}$ & 10 \\
\hline $\mathrm{Li}_{3} \mathrm{YBr}_{6}$ & $1.7 \times 10^{-3}$ & 10 \\
\hline $\mathrm{Li}_{3} \mathrm{ErCl}_{6}$ & $0.17-3.3 \times 10^{-4}$ & 11 \\
\hline $\mathrm{Li}_{3} \mathrm{InCl}_{6}$ & $0.84-2.04 \times 10^{-3}$ & $12-13$ \\
\hline $\mathrm{Li}_{3-\mathrm{x}} \mathrm{M}_{1-\mathrm{x}} \mathrm{ZrxCl}_{6}(\mathrm{M}=\mathrm{Y}, \mathrm{Er})$ & up to $1.4 \times 10^{-3}$ & 14 \\
\hline $\mathrm{Li}_{3} \mathrm{ErI}_{6}$ & $3.9-6.5 \times 10^{-4}$ & 15 \\
\hline $\mathrm{Li}_{2-2 \mathrm{x}} \mathrm{Fe}_{1+\mathrm{x}} \mathrm{Cl}_{4}$ & up to $1.3 \times 10^{-5}$ & 16 \\
\hline $\mathrm{Li}_{2-2 \mathrm{x}} \mathrm{M}_{1+\mathrm{x}} \mathrm{Cl}_{4}(\mathrm{M}=\mathrm{Mg}, \mathrm{Mn}, \mathrm{Cd})$ & up to $1.5 \times 10^{-5}$ & 17 \\
\hline $\mathrm{LiScCl}_{6}$ & $3 \times 10^{-3}$ & 18 \\
\hline
\end{tabular}


Table S2 The ionic radius of tetravalent metal cations in the fifth period (coordination

$$
\text { number }=6)^{19}
$$

\begin{tabular}{|c|c|c|c|c|c|c|c|c|}
\hline Ion & $\mathrm{Zr}^{4+}$ & $\mathrm{Nb}^{4+}$ & $\mathrm{Mo}^{4+}$ & $\mathrm{Tc}^{4+}$ & $\mathrm{Ru}^{4+}$ & $\mathrm{Rh}^{4+}$ & $\mathrm{Pd}^{4+}$ & $\mathrm{Sn}^{4+}$ \\
\hline Radius/pm & 72 & 68 & 65 & 64.5 & 62 & 60 & 61.5 & 69 \\
\hline
\end{tabular}

The cell refinement results by MDI Jade 9 is shown in the table below $(\alpha=\gamma=90$ $\left.{ }^{\circ}\right)$. The cell is refined based on the XRD results in the angle range of $10-80^{\circ}$ and the results are to show the evolution tendency of the structural parameters for these samples on the whole. Its accuracy of the parameters for $\mathrm{Li}_{2.9} \mathrm{In}_{0.9} \mathrm{Zr}_{0.1} \mathrm{Cl}_{6}$ is lower than the XRD Rietveld refinement analysis results based on the data in the angle range of 10-140 ${ }^{\circ}$ showed in Table S5.

Table S3 The cell refinement results for all prepared electrolytes by MDI Jade 9

\begin{tabular}{|c|c|c|c|c|c|}
\hline Samples & $\mathbf{a} / \mathbf{A}$ & $\mathbf{b} / \AA ̊$ & $\mathbf{c} / \AA ̊ \AA$ & $\boldsymbol{\beta} /{ }^{\circ}$ & $\mathbf{V} / \AA^{3}$ \\
\hline PDF\#04-009-9027 & 6.432 & 11.093 & 6.398 & 109.8 & 429.51 \\
\hline $\mathrm{Li}_{3} \mathrm{InCl}_{6}$ & $6.378(1)$ & $11.119(5)$ & $6.371(2)$ & 109.60(4) & 425.73 \\
\hline $\mathrm{Li}_{2.95} \mathrm{In}_{0.95} \mathrm{Zr}_{0.05} \mathrm{Cl}_{6}$ & $6.425(2)$ & $11.083(3)$ & $6.405(2)$ & 109.79(3) & 429.26 \\
\hline $\mathrm{Li}_{2.9} \mathrm{In}_{0.9} \mathrm{Zr}_{0.1} \mathrm{Cl}_{6}$ & $6.430(3)$ & $11.087(4)$ & $6.408(3)$ & 109.83(3) & 429.78 \\
\hline $\mathrm{Li}_{2.85} \mathrm{In}_{0.85} \mathrm{Zr}_{0.15} \mathrm{Cl}_{6}$ & $6.431(5)$ & $11.103(3)$ & $6.384(4)$ & $109.22(6)$ & 430.48 \\
\hline $\mathrm{Li}_{2.8} \mathrm{In}_{0.8} \mathrm{Zr}_{0.2} \mathrm{Cl}_{6}$ & $6.429(2)$ & 11.097(3) & $6.411(3)$ & $109.88(4)$ & 430.21 \\
\hline $\mathrm{Li}_{2.75} \mathrm{In}_{0.75} \mathrm{Zr}_{0.25} \mathrm{Cl}_{6}$ & $6.398(6)$ & $11.099(5)$ & $6.379(4)$ & $109.75(7)$ & 426.4 \\
\hline
\end{tabular}

Table S4 Atomic coordinates, occupation factor and isotropic displacement parameters of $\mathrm{Li}_{3} \mathrm{InCl}_{6}$ obtained from data base (space group C2/m, ICSD No. 04-009-9027) ${ }^{20}, \mathrm{a}=6.43200 \AA, \mathrm{b}=$ $11.09300 \AA, c=6.39800 \AA, \alpha=\gamma=90^{\circ}, \beta=109.8^{\circ}$, Unit-cell volume $=429.510593 \AA^{3}$.

\begin{tabular}{ccccccc}
\hline Atom & $\mathbf{x}$ & $\mathbf{y}$ & $\mathbf{z}$ & OCC & Site & Sym \\
\hline In1 & 0 & 0.3333 & 0 & 0.07 & $4 \mathrm{~g}$ & 2 \\
In2 & 0 & 0 & 0 & 0.875 & $2 \mathrm{a}$ & $2 / \mathrm{m}$ \\
Cl3 & 0.245 & 0 & -0.2338 & 1 & $4 \mathrm{i}$ & $\mathrm{m}$ \\
Li4 & 0.5 & 0 & 0.5 & 1 & $2 \mathrm{~d}$ & $2 / \mathrm{m}$ \\
Li5 & 0 & 0.1683 & 0.5 & 1 & $4 \mathrm{~h}$ & 2 \\
Cl6 & 0.2421 & 0.1622 & 0.2388 & 1 & $8 \mathrm{j}$ & 1 \\
\hline
\end{tabular}

Table S5 Crystallographic data of $\mathrm{Li}_{2.9} \mathrm{In}_{0.9} \mathrm{Zr}_{0.1} \mathrm{Cl}_{6}$ and corresponding refined parameters.

\begin{tabular}{ll}
\hline Compound & $\mathrm{Li}_{2.9} \mathrm{In}_{0.9} \mathrm{Zr}_{0.1} \mathrm{Cl}_{6}$ \\
\hline
\end{tabular}




\begin{tabular}{cc}
\hline Space group & $\mathrm{C} 2 / \mathrm{m}$ \\
$\mathbf{a}, \AA$ & $6.39857 \pm 0.00083$ \\
$\mathbf{b}, \AA$ & $11.10032 \pm 0.00202$ \\
$\mathbf{c}, \AA$ & $6.37649 \pm 0.00107$ \\
$\boldsymbol{\alpha}=\boldsymbol{\gamma},^{\circ}$ & 90 \\
$\boldsymbol{\beta},^{\circ}$ & $109.29373 \pm 0.00759$ \\
$\mathbf{V}, \AA^{\mathbf{3}}$ & 427.4617 \\
$\mathbf{2 \theta}$ interval, & $10-140$ \\
Rwp, $\%^{\circ}$ & 4.33 \\
Rp, \% & 2.99 \\
Radiation & $\mathrm{Cu} \mathrm{k \alpha}, \lambda=1.54178 \AA$ \\
\hline
\end{tabular}

Table S6 XRD Rietveld refinement analysis results of $\mathrm{Li}_{2.9} \mathrm{In}_{0.9} \mathrm{Zr}_{0.1} \mathrm{Cl}_{6}$

\begin{tabular}{ccccccc}
\hline Atom & $\mathbf{x}$ & $\mathbf{y}$ & $\mathbf{z}$ & OCC. & Site & Sym \\
\hline In1 & 0 & 0.3333 & 0 & 0.161 & $4 \mathrm{~g}$ & 2 \\
Zr1 & 0 & 0.3333 & 0 & 0.07 & $4 \mathrm{~g}$ & 2 \\
In2 & 0 & 0 & 0 & 0.577 & $2 \mathrm{a}$ & $2 / \mathrm{m}$ \\
Li4 & 0.5 & 0 & 0.5 & 0.86 & $2 \mathrm{~d}$ & $2 / \mathrm{m}$ \\
Li5 & 0 & 0.16467 & 0.5 & 1 & $4 \mathrm{~h}$ & 2 \\
Cl3 & 0.23973 & 0 & 0.76221 & 1 & $4 \mathrm{i}$ & $\mathrm{m}$ \\
Cl6 & 0.26662 & 0.16914 & 0.23506 & 1 & $8 \mathrm{j}$ & 1 \\
\hline
\end{tabular}

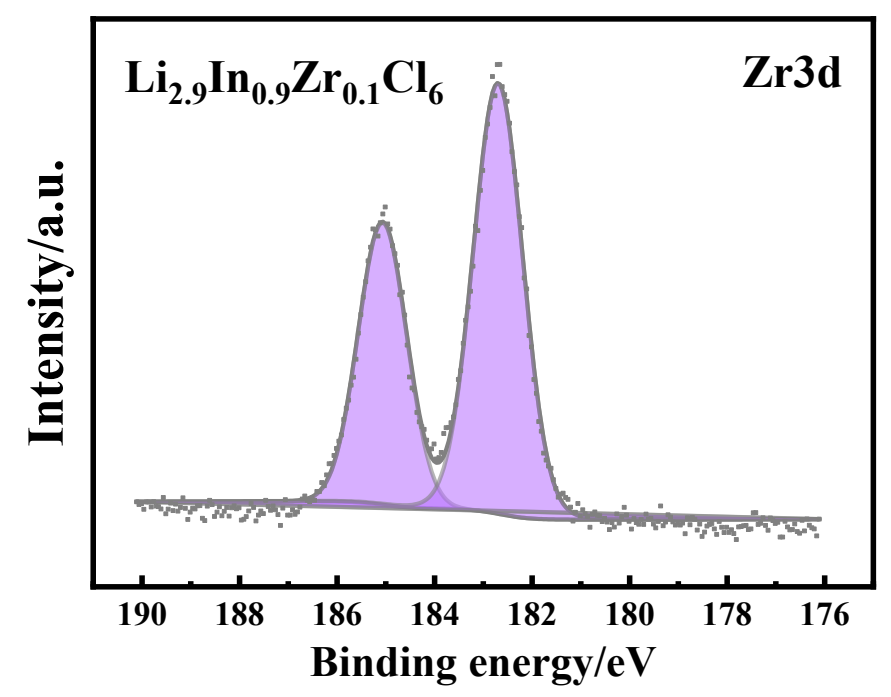

Figure S3 X-ray photoelectron $\mathrm{Zr} 3 \mathrm{~d}$ spectra of as-prepared $\mathrm{Li}_{2.9} \mathrm{In}_{0.9} \mathrm{Zr}_{0.1} \mathrm{Cl}_{6}$ pellet 
Table S7 Summary of the ionic conductivities and corresponding activation energy values of $\mathrm{Li}_{3-}$

$$
{ }_{\mathrm{x}} \mathrm{In}_{1-\mathrm{x}} \mathrm{Zr}_{\mathrm{x}} \mathrm{Cl}_{6}(\mathrm{x}=0,0.05,0.1,0.15,0.2,0.25) \text {. }
$$

\begin{tabular}{ccc}
\hline Samples & Ionic Conductivity $@ \mathbf{~ 2 0}^{\circ} \mathbf{C}\left(\mathbf{m S ~ c m}^{-1}\right)$ & Activation Energy (eV) \\
\hline $\mathrm{Li}_{3} \mathrm{InCl}_{6}$ & 0.88 & 0.320 \\
$\mathrm{Li}_{2.95} \mathrm{In}_{0.95} \mathrm{Zr}_{0.05} \mathrm{Cl}_{6}$ & 0.49 & 0.322 \\
$\mathrm{Li}_{2.9} \mathrm{In}_{0.9} \mathrm{Zr}_{0.1} \mathrm{Cl}_{6}$ & 1.54 & 0.296 \\
$\mathrm{Li}_{2.85} \mathrm{In}_{0.85} \mathrm{Zr}_{0.15} \mathrm{Cl}_{6}$ & 1.26 & 0.307 \\
$\mathrm{Li}_{2.8} \mathrm{In}_{0.8} \mathrm{Zr}_{0.2} \mathrm{Cl}_{6}$ & 0.24 & 0.337 \\
$\mathrm{Li}_{2.75} \mathrm{In}_{0.75} \mathrm{Zr}_{0.25} \mathrm{Cl}_{6}$ & 0.60 & 0.321 \\
\hline
\end{tabular}

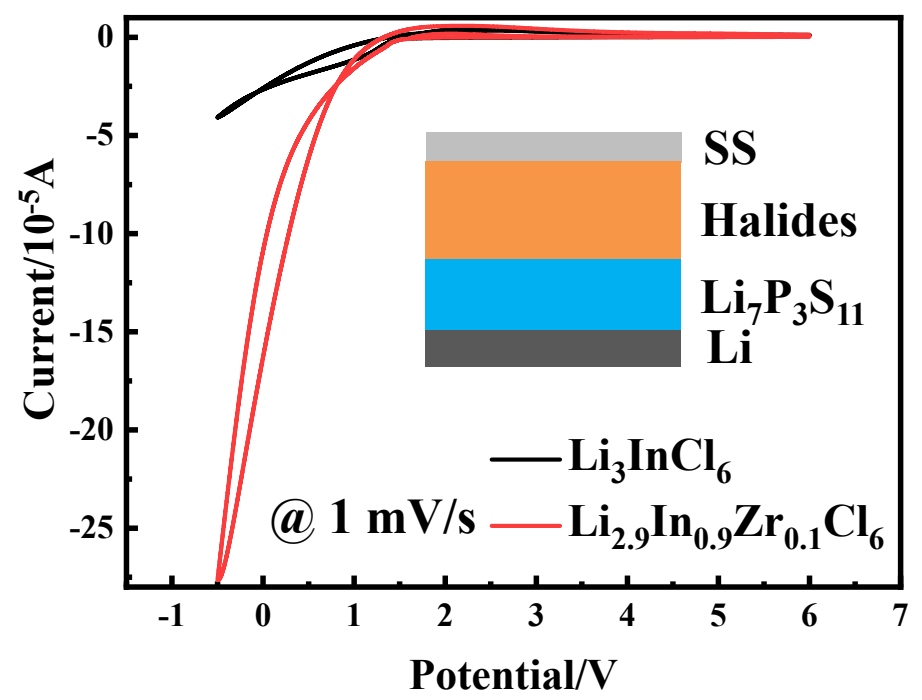

Figure S4 Cyclic voltammogram of original $\mathrm{Li}_{3} \mathrm{InCl}_{6}$ and $\mathrm{Li}_{2.9} \mathrm{In}_{0.9} \mathrm{Zr}_{0.1} \mathrm{Cl}_{6}$.
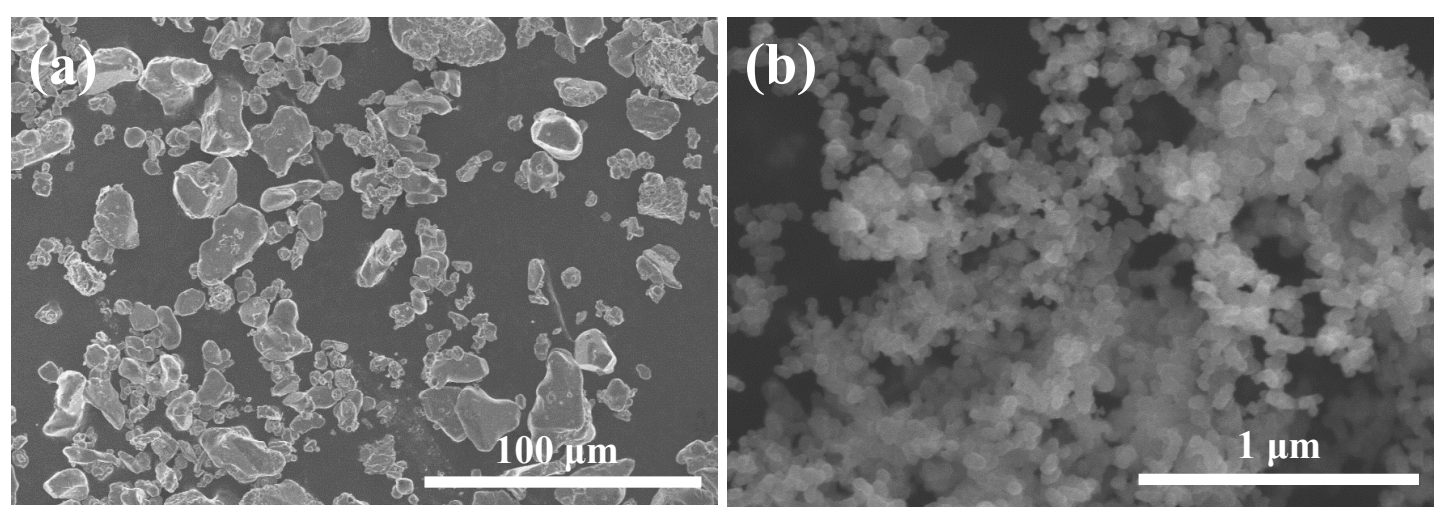

Figure S5 Micro-morphology of (a) $\mathrm{LiCoO}_{2}$ and (b) conductive carbon black used in the ASSLMB-assembling. 


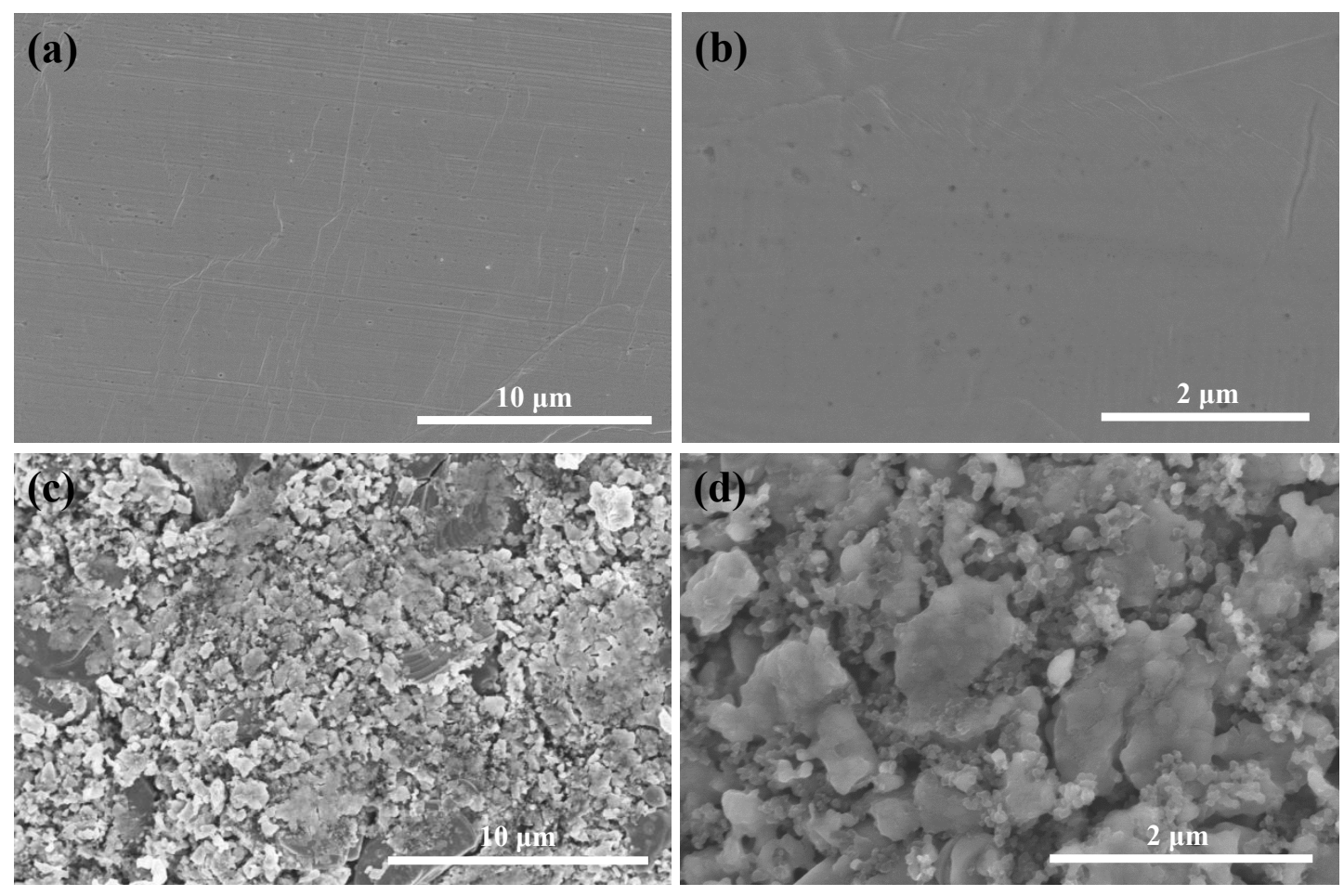

Figure S6 Pristine surface morphology of (a)(b) In foil and (c)(d) composite cathode pellet in ASSLMB based on $\mathrm{Li}_{2.9} \mathrm{In}_{0.9} \mathrm{Zr}_{0.1} \mathrm{Cl}_{6}$ electrolyte before 50 cycles

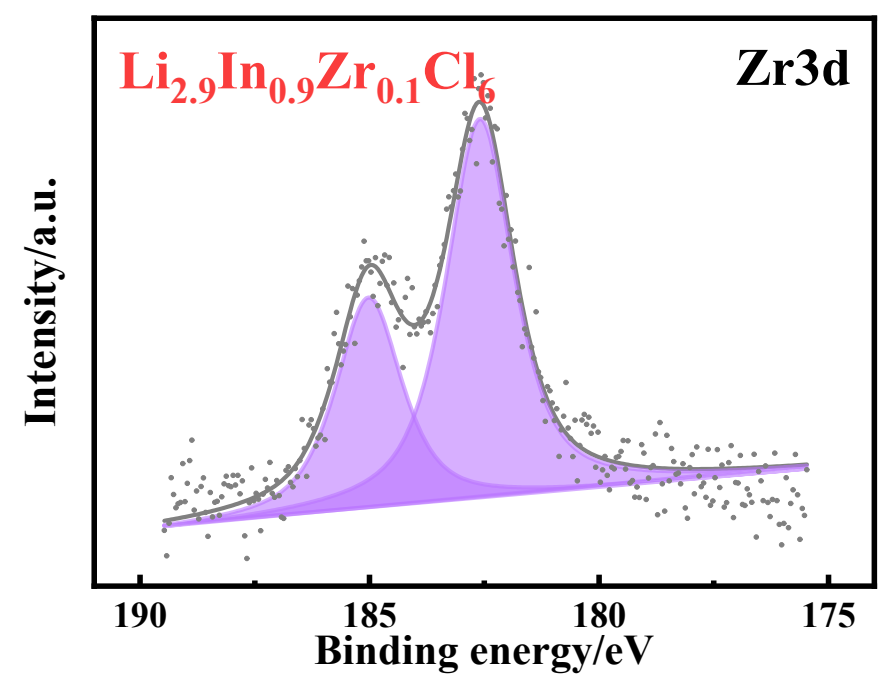

Figure S7 X-ray photoelectron Zr3d of composite cathode pellets in the ASSLMBs based on

$$
\mathrm{Li}_{2.9} \mathrm{In}_{0.9} \mathrm{Zr}_{0.1} \mathrm{Cl}_{6} \text { after } 50 \text { cycles. }
$$

\section{References}

(1) Jackson, B. J. H.; Young, D. A. Ionic Conduction in Pure and Doped Single-Crystalline Lithium Iodide. J Phys Chem Solids 1969, 30, 1973-1976. 
(2) Haven, Y. The Ionic Conductivity of Li-Halide Crystals. Recueil Des Travaux Chimiques Des PaysBas-Journal of the Royal Netherlands Chemical Society 1950, 69, 1471-1489.

(3) Schlaikjer, C. R.; Liang, C. C. Ionic Conduction in Calcium Doped Polycrystalline Lithium Iodide. $J$ Electrochem Soc 1971, 118, 1447-+.

(4) Weppner, W.; Huggins, R. A. Ionic-Conductivity of Alkali-Metal Chloroaluminates. Physics Letters A 1976, 58, 245-248.

(5) Weppner, W.; Huggins, R. A. Ionic-Conductivity of Solid and Liquid LiAlCl4. J Electrochem Soc 1977, 124, 35-38.

(6) Oi, T.; Miyauchi, K. Amorphous Thin-Film Ionic Conductors of mLiF.nAlF3. Mater Res Bull 1981, 16, 1281-1289.

(7) Oi, T. Ionic Conductivity of Amorphous mLiFnMF3 Thin Films (M=Al, Cr, Sc or Al+Sc). Mater Res Bull 1984, 19, 1343-1348.

(8) Esaka, T.; Okuyama, R.; Iwahara, H. Ionic-Conduction in Sintered Fluorocomplexes LimMF6, M=Al, Ti. Solid State Ionics 1989, 34, 201-205.

(9) Tomita, Y.; Fuji-i, A.; Ohki, H.; Yamada, K.; Okuda, T. New Lithium Ion Conductor Li3InBr6 Studied by Li-7 NMR. Chem Lett 1998, 27, 223-224.

(10) Asano, T.; Sakai, A.; Ouchi, S.; Sakaida, M.; Miyazaki, A.; Hasegawa, S. Solid Halide Electrolytes with High Lithium-Ion Conductivity for Application in $4 \mathrm{~V}$ Class Bulk-Type All-Solid-State Batteries. Adv Mater 2018, 30, 1803075.

(11) Muy, S.; Voss, J.; Schlem, R.; Koerver, R.; Sedlmaier, S. J.; Maglia, F.; Lamp, P.; Zeier, W. G.; Shao-Horn, Y. High-Throughput Screening of Solid-State Li-Ion Conductors Using Lattice-Dynamics Descriptors. Iscience 2019, 16, 270-282.

(12) Li, X. N.; Liang, J. W.; Chen, N.; Luo, J.; Adair, K. R.; Wang, C. H.; Banis, M. N.; Sham, T. K.; Zhang, L.; Zhao, S. Q.; Lu, S. G.; Huang, H.; Li, R. Y.; Sun, X. L. Water-Mediated Synthesis of a Superionic Halide Solid Electrolyte. Angew Chem Int Ed Engl 2019, 58, 16427-16432.

(13) Li, X. N.; Liang, J. W.; Luo, J.; Norouzi Banis, M.; Wang, C. H.; Li, W. H.; Deng, S. X.; Yu, C.; Zhao, F. P.; Hu, Y. F.; Sham, T. K.; Zhang, L.; Zhao, S. Q.; Lu, S. G.; Huang, H.; Li, R. Y.; Adair, K. R.; Sun, X. L. Air-Stable Li3InCl6 Electrolyte with High Voltage Compatibility for All-Solid-State Batteries. Energy and Environmental Science 2019, 12, 2665-2671.

(14) Park, K.-H.; Kaup, K.; Assoud, A.; Zhang, Q.; Wu, X.; Nazar, L. F. High-Voltage Superionic Halide Solid Electrolytes for All-Solid-State Li-Ion Batteries. Acs Energy Lett 2020, 5, 533-539.

(15) Schlem, R.; Bernges, T.; Li, C.; Kraft, M. A.; Minafra, N.; Zeier, W. G. Lattice Dynamical Approach for Finding the Lithium Superionic Conductor Li3ErI6. ACS Appl Energ Mater 2020, 3, 3684-3691.

(16) Kanno, R.; Takeda, Y.; Takada, K.; Yamamoto, O. Phase-Diagram and Ionic-Conductivity of the Lithium-Chloride Iron(II) Chloride System. Solid State Ionics 1983, 9-10, 153-156.

(17) Kanno, R.; Takeda, Y.; Takada, K.; Yamamoto, O. Ionic-Conductivity and Phase-Transition of the Spinel System Li2-2xM1+xCl4(M=Mg,Mn,Cd). J Electrochem Soc 1984, 131, 469-474.

(18) Liang, J. W.; Li, X. N.; Wang, S.; Adair, K. R.; Li, W. H.; Zhao, Y.; Wang, C. H.; Hu, Y. F.; Zhang, L.; Zhao, S. Q.; Lu, S. G.; Huang, H.; Li, R. Y.; Mo, Y. F.; Sun, X. L. Site-Occupation-Tuned Superionic LixScCl3+xHalide Solid Electrolytes for All-Solid-State Batteries. J Am Chem Soc 2020, 142, 70127022 .

(19) Shannon, R. D. Revised Effective Ionic-Radii and Systematic Studies of Interatomic Distances in Halides and Chalcogenides. Acta Crystallographica Section A 1976, 32, 751-767.

(20) Schmidt, M. O.; Wickleder, M. S.; Meyer, G. Ternary Halides of the A(3)MX(6) type. VIII - on the 
Crystal Structure of Li3InCl6. Z Anorg Allg Chem 1999, 625, 539-540. 